\title{
Remarks on Neuroptera of Southeastern Turkey
}

\author{
Ali Satar \& Cengizhan Özbay
}

Satar, A. \& Özbay, C. 2004: Remarks on Neuroptera of Southeastern Turkey. Entomol. Fennica 15: 219-224.

The abundance of species of the insect order Neuroptera was studied in the traditionally cultivated landscape of southeastern Turkey. A total of 544 specimens were collected from seven localities during 2000, 2001 and 2002, representing 28 species, 19 genera, and five families of the order. Sixteen species were recorded as new for southeastern Anatolia. Chrysopidae and Myrmeleontidae were the best represented families in the region. Neuroptera occurred both in cultivated fields and natural habitats. Species occurrences were evaluated zoogeographically, and some observations and recommendations were made on the species that could be useful for controlling crop pests.

A. Satar, Department of Biology, University of Dicle 21280 Diyarbakır, Turkey; E-mail: alisatar@dicle.edu.tr

C. Özbay, Department of Biology, University of Dicle 21280 Diyarbakır, Turkey; E-mail:cozbay@dicle.edu.tr

Received 26 June 2003, accepted 9 February 2004

\section{Introduction}

The southeastern part of Turkey is known as one of the best preserved and undamaged natural habitats of Turkey, thanks to the traditional farming methods applied there. The biological diversity is high in the region probably due to the climatic heterogeneity: it is influenced by Eremial, Mediterranian and Siberian climates. Only a few studies of its neuropteran fauna exist (Gerstaecker 1894, Gepp 1974, Şengonca 1979, Şengonca 1980a, Şengonca 1981a, b), but those indicate a high species diversity. For example, thirteen species of Neuropteridae alone have been reported to exist in the study area of the present paper. The aim of our study was, therefore, to provide information on the presence and distribution of different Neuroptera in the southeastern Turkey.

\section{Material and methods}

Neuroptera from seven localities (Fig. 1) were obtained from May to September in 2000, 2001 and 2002. The localities (all within $37^{\circ} 42^{\prime}-43^{\circ}$ $33^{\prime} \mathrm{E}, 36^{\circ} 40^{\prime}-38^{\circ} 42^{\prime} \mathrm{N}$ ) included Adiyaman (site nr. 1), Batman (2), Diyarbakır (3), Mardin (4), Siirt (5), Şanlıurfa (6) and Şırnak (7). The region as a whole is characterized by Iran-Turan steppe biome (Saya \& Ertekin 1997). Three sites (nrs. 1, 5, 7) represented natural habitat of vegetation typical for southeastern Turkey, viz. forests predominated by Quercus infectoria, $Q$. brantii, $Q$. libani and $Q$. cedrorum. Four other sites (nrs. 2, 3, 4 and 6) were on agricultural land such as meadows located between fields.

We collected larvae and adults twice a month using net, beating branches of bushes and trees over an umbrella, hand-collecting larvae, and light-trapped specimens at night. 


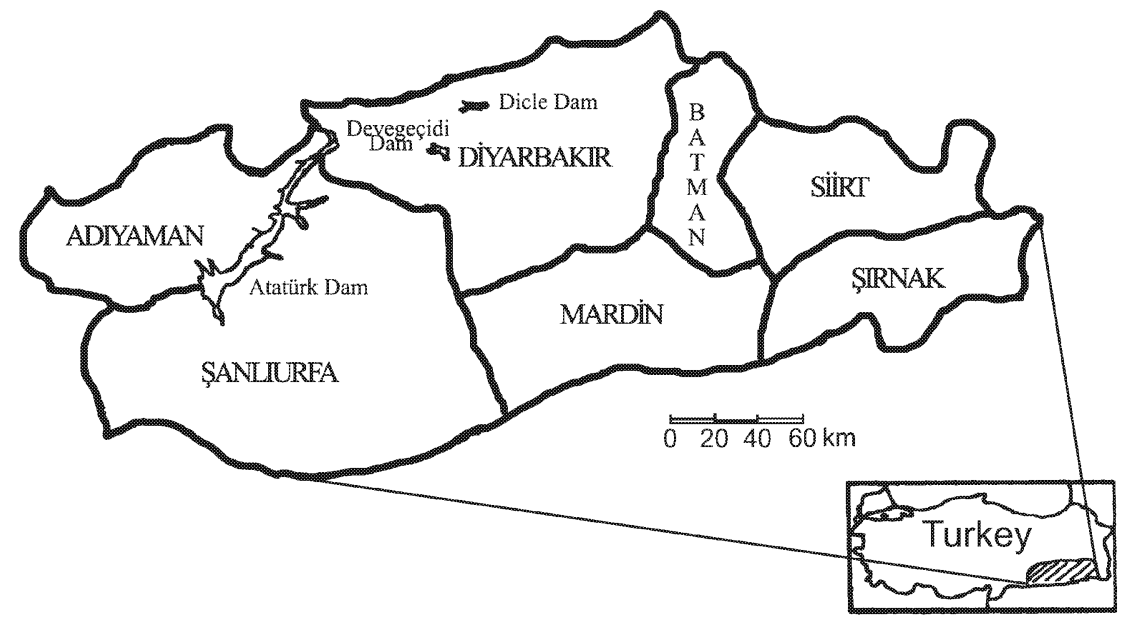

Fig. 1. The collecting sites.

\section{Results}

Twenty-eight species from 19 genera were identified, representing the families Chrysopidae (4 genera, 6 species), Myrmeleontidae (10 genera, 15 species), Nemopteridae ( 3 genera, 4 species), Ascalaphidae (1 genus, 2 species), and Mantispidae (1 genus, 1 species). Some of these species have also been shown to be present in other parts of Turkey, e.g., see Aspöck et al. (1976, 1978, 1980, 1984, 2001), Canbulat (1998, 2000), Demirsoy (1990), Hölzel et al. (1967, 1968, 1972), Satar (2002), and Şengonca (1979, 1980a, b, $1981 \mathrm{a}, \mathrm{b}, \mathrm{c})$. The species and their localities are as follows.

\subsection{Chrysopidae Schneider, 1851}

Italochrysa italica (Rossi, 1970)

Material. 2 21.X.2001, AdiyamanMerkez, 800 ma.s.l.; 1 9, 21.X.2001, AdiyamanBesni, $805 \mathrm{~m}$ a.s.1.. Holo-Mediterrenian faunal element.

Chrysoperla carnea (Stephens, 1836) sensu lato Material. 2 17.VI.2000, ŞanluurfaAkçakale, 450 m a.s.l.; 3 ㅇ 5 13.VI.2000, Batman-BeŞiri, $950 \mathrm{~m} ; 2$ q 1 oे 22.VI.2000, Diyarbakır-Kocaköy, $930 \mathrm{~m}$ a.s.l.; 3 क 2 ठ 15.VII.2000, Adiyaman-Gerger, $1200 \mathrm{~m}$ a.s.l.; 4 I 2 16.VIII.2000, Mardin-YeŞilli; 592 ठ 26.V.2001, Şırnak-Merkez; 4 \& 1 O 08.VI.2002,
Mardin-Nusaybin, $440 \mathrm{~m}$ a.s.l.; $392 \hat{\delta}$ 09.VI.2002, Siirt-Eruh, $1650 \mathrm{~m}$ a.s.l.; 5 q 2 ठ 09.VI.2001, Siirt-Pervari, $1450 \mathrm{~m}$ a.s.1..

Note. Şengonca (1980a) previously reported this species from Diyarbakır Mardin, Siirt and Şanllurfa province in 1976. SiberianMediterranian faunal element.

\section{Chrysopa dubitans McLachlan, 1887}

Material. 2 o 1 o 19.IX.2001, MardinYeşilli; 3 † 2 đ 02.VII.2002, Mardin-Savur, $1100 \mathrm{~m}$ a.s.l.

Note. Şengonca (1980a) previously reported this species from Mardin and Siirt province in 1976. Policentric Eremial faunal element.

Dichocrysa venosa (Rambur, 1842)

Material. 1 9 12.VI.2002, Diyarbakır-Dicle, $930 \mathrm{~m}$ a.s.l..

Note. Gepp (1974) previously reported this species from Mardin province in 1974. Eremial faunal element.

\section{Dichocrysa flavifrons (Brauer, 1850)}

Material. 3 \% 22.VI.2000, DiyarbakirKocaköy, $930 \mathrm{~m}$ a.s.1.; 2 \& 1 సै 15.VII.2000, Adiyaman-Merkez, $720 \mathrm{~m}$ a.s.1.; 3 क $2 \hat{\delta}$ 16.VIII.2000, Mardin-YeŞilli; 2 ㅇ 3 ह 26.V.2001, Şırnak-Merkez; 3 q 2 § 19.IX.2001, Mardin-Savur; 5 q 2 Ỏ 08.VI.2002, Mardin-Sultan köy; 2 2 đ 09.VI.2002, Siirt-Eruh, $1650 \mathrm{~m}$ a.s.1.; 2 오 3 09.VI.2001, Siirt-Pervari, $1800 \mathrm{~m}$ a.s.1. 
Note. Şengonca (1980a) previously reported this species from Mardin province in 1976. HoloMediterrenian faunal element.

Dichocrysa prasina (Burmeister, 1839)

Material. 2 \& 1 o 13.VI.2000, BatmanBeŞiri, 1000 m; 2 + 1 22.VI.2000, DiyarbakırKulp, $1200 \mathrm{~m}$ a.s.1.; 1 q 3 ô 15.VII.2000, Adiyaman-Gerger, $900 \mathrm{~m}$ a.s.l.; 2 † 2 ठ 16.VIII.2000, Mardin-Savur, 1020 m a.s.l.; 2 \& 3 o 19.IV.2001, Mardin-Sultan köy; 2 † 2 ઈิ 26.V.2001, Şırnak-Merkez; 5 \& 2 đ̊ 09.VI.2001, Siirt-Pervari, $1450 \mathrm{~m}$ a.s.1.; 2 † 3 ठ08.V.2002, Mardin-BeŞ Evler; 2 + 3 ठ 08.VI.2002, MardinNusaybin, 430 m a.s.1.; 3 \& 2 09.VI.2002, Şanliurfa-Siverek, $900 \mathrm{~m}$ a.s.l..

Note. Şengonca (1980a) previously reported this species from Mardin province in 1976. Siberian-Mediterranian faunal element.

\subsection{Myrmeleontidae Latreille, 1803}

Palpares libelluloides (Linnaeus, 1767)

Material. 3 \& 1 of 13.VI.2000, Siirt-Pervari, $1450 \mathrm{~m}$ a.s.1.; 2 \& 1 § 15.IX.2000, DiyarbakırÇınar, 714 m a.s.l.; 1 o 1 ठै 15.IX.2000, ŞırnakMerkez; 1 q 2 o 20.V.2001, Batman-BeŞiri, 1120 m a.s.1.; 1 q 26.V.2001, Siirt-Eruh, $1680 \mathrm{~m}$ a.s.l.; 3 \& 1 14.IX.2001, Adryaman-Merkez, $750 \mathrm{~m}$ a.s.l.; 1 † 2 12.VI.2002, DiyarbakırDicle, 1135 m a.s.1.; 3 \& 1 0 08.VI.2002, SiirtPervari, $1450 \mathrm{~m}$ a.s.l.; 2 † 2 §े 09.VI.2002, Şanlıurfa-Hilvan, $800 \mathrm{~m}$ a.s.l..

Note. Holo-Mediterrenian faunal element.

Acanthaclisis occitanica (Villers, 1789)

Material. 1 + 14.IX.2001, AdryamanGerger, $1030 \mathrm{~m}$ a.s.1..

Note. Holo-Mediterrenian faunal element.

Myrmeleon inconspicuus Rambur, 1842

Material. 2 o 1 o 10.VIII.2000, DiyarbakırÇermik; 3 q 14.IX.2001, Adıyaman-Gerger, 1150 m a.s.1.; 1 \& 2 क 21.X.2001, AdiyamanBesni, $700 \mathrm{~m}$ a.s.l.; 1 q 2 ô 14.VIII.2002, Diyarbakır-Merkez, 695 m a.s.l..

Note. Holo-Mediterrenian faunal element.
Myrmecaelurus trigrammus (Palas, 1781)

Material. 2 3 o 13.VI.2000, BatmanBeŞiri, $800 \mathrm{~m}$ a.s.l.; 2 o 1 o 13.VI.2000, Batman-Kurtalan, 700 m a.s.1.; 3 \& 1 15.IX.2000, Şırnak-Merkez; 2 q 1 đ 08.VI.2002, MardinNusaybin, $450 \mathrm{~m}$ a.s.l.; 1 † 2 है 12.VI.2002, Diyarbakır-Merkez, $700 \mathrm{~m}$ a.s.l.; 3 \% 02.VII.2002, Mardin-Savur, $1000 \mathrm{~m}$ a.s.1..

Note. Holo-Mediterrenian faunal element.

Myrmecaelurus maior McLachlan, 1875

Material. 2 3 ₹ 15.VII.2000, Adıyaman-

Gerger, 1200 m a.s.1.; 1 † 19.VII.2002,

Diyarbakır-Ergani, $890 \mathrm{~m}$ a.s.l..

Note. Irano-Eremial faunal element.

Solter ledereri Navás, 1912

Material. 1 ठ 15.0IX.2000, DiyarbakırÇınar; $720 \mathrm{~m}$ a.s.l.; 1 \% 18.X.2000, MardinÖmerli, 1050 m a.s.1.; $2 \precsim 21 . X .2001$, AdıyamanMerkez, $760 \mathrm{~m}$ a.s.l..

Note. Syro-Eremial faunal element.

Cueta lineosa (Rambur, 1842)

Material. 1 of 15.VII.2000, AdiyamanGerger, 970 m a.s.1.; 2 27.V.2001, Adıyaman, 1 o 1 of 29.V.2002, Diyarbakır-Çermik.

Note. Eremial faunal element.

Neuroleon assimilis Navás, 1914

Material. 2 o 1 ô 11.V.2000, DiyarbakırMerkez, 769m a.s.1.; 3 q 13.VI.2000, BatmanMerkez, 770 m a.s.l.; 1 o 13.VI.2000, BatmanKurtalan, 700 m a.s.l.; 4 \& 1 ઈ 08. VI.2002, Mardin-Kiziltepe, 435m a.s.l.; 2 12.VI.2002, Diyarbakır-Merkez, $690 \mathrm{~m}$ a.s.l..

Note. Ponto-Mediterranean faunal element.

Neuroleon tenellus (Klug, 1834)

Material. 4 \& 2 ô 11.V.2000, DiyarbakırMerkez, $750 \mathrm{~m}$ a.s.1.; 2 \& $2 \delta$ 13.VI.2000, Batman-Merkez, 735m a.s.l.; 3 q 1 f 13.VI.2000, Batman-Kurtalan, $700 \mathrm{~m}$ a.s.1.; 2 क $1 \delta$ 08.VI.2002, Mardin-Nusaybin, $430 \mathrm{~m}$ a.s.l.; 2 ㅇ 12.VI.2002, Diyarbakir-Merkez, $769 \mathrm{~m}$ a.s.1..

Note. Şengonca (1979) previously reported this species from Siirt province in 1976. AfroIrano-Eremial faunal element. 
Neuroleon egenus (Navás, 1915)

Material. 1 \& 11.V.2001, Diyarbakır-

Merkez, $690 \mathrm{~m}$ a.s.1.; 1 q 20.V.2001, Batman-

BeŞiri, 1000 m a.s.l.; 1 q 27.V.2001, Adıyaman-

Besni, $825 \mathrm{~m}$ a.s.l..

Note. Holo-Mediterranean faunal element.

Neuroleon microstenus (McLachlan, 1898)

Material. 2 † 2 ठ 09.VI.2001, DiyarbakırMerkez; 1 \& 2 of 13.VI.2001, Diyarbakır-Lice, $1100 \mathrm{~m}$ a.s.1.; 3 o 2 ô 08.VI.2002, MardinNusaybin, $430 \mathrm{~m}$ a.s.1..

Note. Irano-Eremial faunal element.

Creoleon plumbeus (Oliver, 1811)

Material. 2 o 1 đे 13.VI.2000, Batman-

Kurtalan, 700 m a.s.1.; 3 13.VI.2000, Batman-

Merkez, $800 \mathrm{~m}$ a.s.1.; 3 q 2 o 15.IX.2000, Şırnak-Merkez; 2 ㅇ 1 17.VI.2001, ŞanlıurfaMerkez, $700 \mathrm{~m}$ a.s.1.; 2 + 3 ठै 14.IX.2001, Adiyaman-Gerger, $1050 \mathrm{~m}$ a.s.l.; 1 \& 1 d 08.VI.2002, Mardin-Nusaybin, 430 m a.s.l.; 2 ㅇ $2 \lambda$ 06.IX.2002, Siirt-Eruh, 1680 ma.s.l.; 1 ㅇ $2 \lambda$ 12.VI.2002, Diyarbakır-Merkez, $718 \mathrm{~m}$ a.s.l..

Note. Şengonca (1979) previously reported this species from Sanlurfa province in 1976. Ponto-Mediterranean faunal element.

\section{Distoleon tetragrammicus (Fabricius, 1798)} Material. 1 \& 1 \& 11.V.2000, DiyarbakırMerkez, 700 m a.s.l.; 1 ô 25.V.2000, DiyarbakırKulp, 1200 m a.s.l.; 1 q 2 ô 25.V.2000, Diyarbakır-Eski Lice; 1 q 15.IX.2000, ŞırnakMerkez; 1 \& 1 ò 13.VI.2001, DiyarbakırKocaköy, 930 m a.s.l.; 1 q 1 ^ 08.VI.2002, Şırnak-Cizre; 2 1 ภ̊ 08.VI.2002, Şırnak-Silopi; 1 o 2 ô 12.VI.2002, Diyarbakır-Merkez, 705m a.s.l..

Note. Holo-Mediterranean faunal element.

Distoleon curdicus Hölzel, 1972

Material. 1 त 25.V.2000, Diyarbakır-Kulp, 1150 m a.s.1.; 1 + 15.VII.2000, AdıyamanGerger, 1020 m a.s.1.; 1 \& 1 ઈै 13.VI.2001, Diyarbakır-Lice, $1200 \mathrm{~m}$ a.s.l.; 1 क 2 12.VI. 2002, Diyarbakır-Merkez, 695m a.s.1.; 1 q 19.VII.2002, Diyarbakır-Ergani, $1100 \mathrm{~m}$ a.s.1.. Note. Syro-Eremial faunal element.
Nedroledon striatus (Klug, 1834)

Material. 1 † 25.V.2000, DiyarbakırKocaköy, 930 m a.s.1..

Note. Irano-Eremial faunal element.

\subsection{Ascalaphidae Rambur, 1842}

Bubopsis hamata Klug, 1834

Material. 2 † 16.VIII.2000, Mardin-YeŞilli; 1 §ै 18.X.2000, Mardin-Ömerli, 1025m a.s.l.; 1 đ13.VI.2001, Diyarbakır-Merkez, 725m a.s.l.; 2 q 13.VI.2002, Siirt-Pervari, $1800 \mathrm{~m}$ a.s.1..

Note. Şengonca (1979) previously reported this species from Sanluurfa province in 1976. Syro-Eremial faunal element.

Bubopsis zarudnyi Alexandrova-Martynova, 1926

Material. 2 9 17.VII.2002, Diyarbakır, Karacadağ Mountain, $930 \mathrm{~m}$ a.s.1..

Note. Satar (2002) previously reported this species from Diyarbakır province in 2002. IranoEremial faunal element.

\subsection{Nemopteridae Burmeister, 1839}

Nemoptera sinuata Oliver, 1811

Material. 1 \& 13.VII.2000, ŞanliurfaCeylanpınar, $436 \mathrm{~m}$ a.s.1.; 1 \& 2 ô 09.VII.2001, Şanlıurfa-Ceylanpınar, $420 \mathrm{~m}$ a.s.1..

Note. Şengonca (1981b) previously reported this species from Şanliurfa province in 1981. Ponto-Mediterranean faunal element.

Lertha extensa (Oliver, 1811)

Material. 10 \& 8 09.VI.2001, DiyarbakırMerkez, $690 \mathrm{~m}$ a.s.l.; 1 \% 13.VI.2000, Karacadağ, 1150 m a.s.l.; 2 \& 1 13.VI.2001, Diyarbak1r-Lice, $1100 \mathrm{~m}$ a.s.l.; 4 † $3 \hat{\delta}$ 08.VI.2002, Mardin-Nusaybin, $435 \mathrm{~m}$ a.s.l.; 20 ㅇ 27 ô 09.VI.2002, Şırnak-Merkez; 1 + 2 ठ 09.VI.2002, Siirt-Kozluk, 800 m a.s.1.; 2 \& 3 09.VI.2002, Siirt-Eruh, $920 \mathrm{~m}$ a.s.1.; 1 \& 1 ठ 10.VI.2002, Siirt-Baykan, $930 \mathrm{~m}$ a.s.l.

Note. Şengonca (1981b) previously reported this species from Şanlıurfa province in 1981. Syro-Eremial faunal element. 
Lertha sheppardi (Kirby, 1904)

Material. 1 \& 7 o 17.VI.2000 DiyarbakırBismil; 2 † 8 đ̊ 9.V.2001, Şanlıurfa-Siverek, 950 m a.s.1.; 10 † 7 09.VI.2001, Karacadağ, 1150 m a.s.1.; 30 q 18 ô 09.VI.2002, DiyarbakırMerkez, $710 \mathrm{~m}$ a.s.l.; 17 q 13 o 09.VI.2002, Karacadağ, $1150 \mathrm{~m}$ a.s.l..

Note. Eremial faunal element.

Dielocroce ephemera (Gerstäcker, 1894)

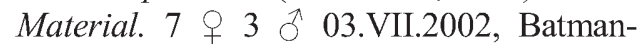
BeŞiri, $1020 \mathrm{~m}$ a.s.l..

Note. Gerstaecker (1894) previously reported this species from Mardin province in 1894. IranoEremial faunal element.

\subsection{Mantispidae Leach in Brewster, 1815}

\author{
Mantispa scabricollis McLachlan, 1875 \\ Material. 2 of 02.VII.2002, Mardin-Savur, \\ $1100 \mathrm{~m}$ a.s.l.. \\ Note. Ponto-Mediterranean faunal element.
}

\section{Discussion}

Of the 28 species identified here, 16 were new for southeastern Anatolia: Italochrysa italica, Palpares libelluloides, Acanthaclisis occitanica, Myrmeleon inconspicuus, Myrmecaelurus trigrammus, Myrmecaelurus maior, Solter ledereri, Cueta lineosa, Neuroleon assimilis, Neuroleon egenus, Neuroleon microstenus, Distoleon tetragrammicus, Distoleon curdicus, Nedroledon striatus, Lertha sheppardi and Mantispa scabricollis. Families Chrysopidae and Myrmeleontidae are well represented in the region, with species found both in cultivated fields and in "natural" habitats. Because the larvae of most neuropteran families feed largely on soft-bodied herbivorous insects and mites (e.g. Stange 1980, Stelzl \& Devetak 1999), keeping the vegetation structure of the surroundings of neuropteran habitat in good condition is an excellent way of controlling insect crop pests (Stelzl \& Devetak 1999).

A part of the Eremial region, southeastern Turkey hosts particularly high biological diversity due to its disparate climate. Mediterranean conditions are found in the southern and southeastern Turkey, while Siberian climate characterizes northern and northeastern regions (Demirsoy 1996). We hope that this study of the neuropteran fauna of southeastern Turkey, together with existing and future faunal works on other groups of plants and animals living in this region, will help to elucidate broader zoogeographic features of this area.

Acknowledgements. We thank Prof. Charles S. Henry, the Department of Ecology and Evolutionary Biology, University of Connecticut, who kindly consented to read the manuscript for linguistic improvements.

\section{References}

Aspöck, H., Aspöck, U. \& Şengonca, Ç. 1976: Raphidia (Phidiara) ramane n. sp., eine neue Kamelhalsfliege aus Vorderasien (Neur., Raphidioptera, Raphidiidae). -Zschr. Arbgem. Österr. Ent. 28: 14-16.

Aspöck, H., Aspöck, U. \& Şengonca, Ç. 1978: Raphidia (Ornatoraphidia) marielouisae n. sp., eine neue Kamelhalsfliege aus Südanatolien (Neuropteroidea: Raphidioptera). - Entom. Zeitschr. (Stuttgart) 88: 165-168.

Aspöck, H., Aspöck, U. \& Hölzel, H. 1980: Die Neuropteren Europas. Eine Zusammenfassende Darstellung der Systematik, Ökologie und Chorologie der Neuropteridae (Megaloptera, Raphidioptera, Planipennia) Europas. - Goecke \& Evers, Krefeld. $495+$ $355 \mathrm{pp}$.

Aspöck, H., Aspöck, U. \& Hölzel, H. 1984: Neue Spezies der Genera Kirbynia Navás und Lertha aus Vorderasien und bemerkungen über Oliverina extensa (Olivier) (Neuropteroidae: Planipennia: Nemopteridae). - Ent. Z. Frankfurt am Main 94: 113-121.

Aspöck, H., Hölzel, H. \& Aspöck, U. 2001: Denisia 2. Kommentierter katalog der Neuropterida (Insecta: Raphidioptera, Megaloptera, Neuroptera) der Westpaläearktis. - Landes museum. 612 pp.

Canbulat, S. 1998: Çanakkale Planıpennia (Insecta: Neuropteroidea) türlerininin sistematik ve faunistik yönden incelenmesi. - M. Sc. Thesis, University of Gazi. [In Turkish].

Canbulat, S. \& Kiyak, S. 2000: On the faunistic and systematical studies of Chryosopidae (Insecta: Neuropteroidea: Planipennia) species of Çanakkale province. - Gazi Üniv. Fen Bilimleri Ens. Derg. 12: 1037 1045. [In Turkish].

Demirsoy, A. 1990: Yaşamın Temel Kurallari. Entomoloji. - Meteksan Matbaacılık, Ankara. 941 pp. [In Turkish].

Demirsoy, A. 1996: Genel ve Türkiye Zoocoğrafyası. Meteksan Matbaacılık, Ankara. 630 pp. [In Turkish].

Gepp, J. 1974: Beitrag zur Kenntnis der Neuropteren der 
Türkei. - Entom. Ber. (Amsterdam) 34: 102-104.

Gerstaecker, A. 1894: Über neue und weniger bekannte Neuropteren aus der Familie Megaloptera Burm. Mitteilungen des Naturwissenschaftlichen Vereins für Neue Vorpommern und Rügen 25: 93-173.

Hölzel, H. 1967: Die Neuropteren Vorderasiens II. Chrysopidae. - Beitr. Naturk. Forsch. Südw. Dtl. 26: 1945.

Hölzel, H. 1968: Die Neuropteren Vorderasiens III. Nemopteridae. - Beitr. Naturk. Forsch. Südw. Dtl. 27: 37-47.

Hölzel, H. 1972: Die Neuropteren Vorderasiens IV. Myrmeleonidae. - Beitr. Naturk. Forsch. Südw. Dtl. 1: 3-103.

Satar, A. \& Özbay, C. 2002: Bubopsis zarudnyi Alexandrova-Martynova, $1926 \mathrm{New}$ to Turkey (Neuroptera: Ascalaphıdae), - Boletin De La S. E. A. 31: 192.

Saya, Ö. \& Ertekin, S. 1997: GAP'ın bölge florasına etkileri. Gap'ın ekolojiye ve tarıma etkileri. - Türkiye Cevre Vakfi Yayını. 39-55. [In Turkish].

Stange, L.A. 1980: The ant-lions of Florida. I. Genera (Neuroptera: Myrmeleontidae). - Entomology Circular 215:4.

Stelzl, M. \& Devetak, D. 1999: Neuroptera in agricultural ecosystems. - Agr. Ecosyst. Environ. 74: 305-321.
Şengonca, Ç. 1978: Berotha fulva (Costa 1855) neu für die Türkei (Planipennia, Berothidae). - Türk. Bit. Kor. Derg. 2: 103-106.

Şengonca, C.. 1979: Beitrag zur Neuropterenfauna der Türkei. Nachrbl. Bayer. Ent. 28: 10-15.

Şengonca, Ç. 1980a: Türkiye Chrysopidae (Neuroptera) faunas1 üzerinde sistematik ve taksonomik araştırmalar. - Gida-Tarım ve Hayvancılık Bakanlığı, Zir. Müc. Zir. Kar. Gn. Md. Yayınları, Ankara. 138 pp. [In Turkish].

Şengonca, Ç. 1980b: Türkiye Mantispidae (Insecta: Neuroptera) faunası üzerinde sistematik ve taksonomik araştırmalar. Tübitak VII. Bilim Kongresi, TBAG Biyoloji Seksiyonu, 6-10 Ekim. KuŞadası-Aydın. Tübitak Yayınları 545: 457-473. [In Turkish].

Şengonca, Ç. 1981a: Die Neuropteren Anatoliens. I. Chrysopidae. - Mitt. Münch. Ent. Ges. 71: 121-137. [In Turkish].

Şengonca, Ç. 1981b: Türkiye Nemopteridae (Insecta: Neuroptera) Faunası Üzerine Taksonomik Araştırmalar. II. Faunistik. - Türk. Bit. Kor. Derg. 5: 101114. [In Turkish].

Şengonca, Ç. 1981c: Türkiye Nemopteridae (Insecta: Neuroptera) faunası üzerine taksonomik araŞtşrmalar. I. familyanın genel tanımı. - Türk. Bit. Kor. Derg. 5: 91-99. [In Turkish]. 